\title{
Conserved charge fluctuations using the $D$-measure in heavy-ion collisions
}

\author{
D. K. Mishra, ${ }^{1, \text { A P. K. Netrakanti, }}{ }^{1}$ and P. Garg $^{2}$ \\ ${ }^{1}$ Nuclear Physics Division, Bhabha Atomic Research Center, Mumbai 400085, India \\ ${ }^{2}$ Department of Physics and Astronomy, Stony Brook University, \\ SUNY, Stony Brook, New York 11794-3800, USA
}

\begin{abstract}
We study the net-charge fluctuations, $D$-measure variable, in high energy heavy-ion collisions in heavy-ion jet interaction generator (HIJING), ultrarelativistic quantum molecular dynamics (UrQMD) and hadron resonance gas (HRG) models for various center-of-mass energies $\left(\sqrt{s_{N N}}\right)$. The effect of kinematic acceptance and resonance decay, in the pseudorapidity acceptance interval $(\Delta \eta)$ and lower transverse momentum $\left(p_{T}^{\min }\right)$ threshold, on fluctuation measures are discussed. A strong dependence of $D$ with the $\Delta \eta$ in HIJING and UrQMD models is observed as opposed to results obtained from the HRG model. The dissipation of fluctuation signal is estimated by fitting the $D$-measure as a function of the $\Delta \eta$. An extrapolated function for higher $\Delta \eta$ values at lower $\sqrt{s_{N N}}$ is different from the results obtained from models. Particle species dependence of $D$ and the effect of the $p_{T}^{\text {min }}$ selection threshold are discussed in HIJING and HRG models. The comparison of $D$, at midrapidity, of net-charge fluctuations at various $\sqrt{s_{N N}}$ obtained from the models with the data from the ALICE experiment is discussed. The results from the present work as a function of $\Delta \eta$ and $\sqrt{s_{N N}}$ will provide a baseline for comparison to experimental measurements.

PACS numbers: 25.75.Gz,12.38.Mh,21.65.Qr,25.75.-q,25.75.Nq
\end{abstract}

\section{INTRODUCTION}

One of the major goals of the heavy-ion collisions is to study the phase structure of the quantum chromodynamic (QCD) phase diagram at finite temperature $(T)$ and baryon chemical potential $\left(\mu_{B}\right)$ [1]. Several studies suggest that, at low $T$ and large $\mu_{B}$, a first-order phase transition occurs from quark-gluon-plasma (QGP) to the hadronic phase. At high $T$ and low $\mu_{B}$, there is a cross over phase transition from QGP to the hadron phase [26]. The study of event-by-event fluctuations provides a unique opportunity to describe the thermodynamic properties of the system created in heavy-ion collisions [7 11].

Event-by-event fluctuations of conserved quantities such as net-baryon number, net-electric charge and netstrangeness are proposed as a possible signal of the QGP formation and quark-hadron phase transition [8, 9]. Earlier studies suggest that, enhanced multiplicity fluctuations are connected to the production of QGP droplets, and suppression of fluctuations may occur due to the large difference in the degrees of freedom between the QGP and hadron gas phases [8, 9, 12]. In the QGP phase, quarks with fractional unit charge $1 / 3$ are the charge carriers, while in the hadronic phase hadrons are the charge carriers with unit charges. Hence, net-charge fluctuations in the QGP phase are significantly smaller as compared to those of the hadron phase. These differences may be exploited as indicators of the formation of quark-gluon plasma in high-energy heavy-ion collisions. Thus, the net-charge fluctuations are strongly dependent on the phase of their origin. Due to the rapid expansion of the fireball created in the heavy-ion collisions, the *Electronic address: dkmishra@rcf.rhic.bnl.gov, dkmishra@barc.gov.in of positive $\left(N_{+}\right)$and negative $\left(N_{-}\right)$particles. The vari- fluctuations created in the initial state may survive until the freeze-out 8]. The fluctuations of the net-charge depend on the square of the charge states present in the system. The net-charge fluctuation is plagued by uncertainties due to volume fluctuation, resonance decay, exact local charge conservation, or repulsive forces among hadrons [13]. Experimentally, only a fraction of the particles are measured in the detector acceptance, which may be subject to charge conservation effects. Also the measured fluctuations depend on the survival probability of the charge fluctuations during the hadronization process. The conservation laws limit the dissipation of the fluctuations which suffer after the hadronization has occurred. This dissipation occurs by diffusion. The heavy-ion collisions forming QGP that hadronizes at a time $\tau_{0}$, produces anomalous dynamic charge fluctuations [14]. The hadronic diffusion from $\tau_{0}$ to a freeze-out time $\tau_{f}$ can dissipate these fluctuations. It is observed that, there is a decrease in dynamical charge fluctuation as a function of $\tau_{f}$. Due to the diffusion of particles in rapidity space, these fluctuations may also get diluted in the expanding medium 14, 15]. It is argued that, the reduction of the fluctuation in the QGP phase might be observed only if the fluctuations are measured over a large rapidity range [15]. The QGP suppression of the charge fluctuation is not observed in the experimental data, however the data are consistent with the diffusion estimates. The effect of the critical fluctuations is crucially different from the QGP suppression. While the QGP suppression is the history effect, the critical fluctuations are the equilibrium fluctuations pertaining to the freeze-out point, and the diffusion is necessary to establish them [16].

In the heavy-ion experiments, the collision volume is not directly measured, hence one can get rid of the volume, to first order by taking the ratios of the number 
ance of the ratio of positive and negative particles scaled by the total number of charged particles is defined as the $D$-measure $(D)$, of the net-charge, which provides a measure of charge fluctuations per unit entropy. The $D$ is related to the ratio $R\left(=N_{+} / N_{-}\right)$as $[17,18]$ :

$$
\begin{aligned}
D=\left\langle N_{\mathrm{ch}}\right\rangle\left\langle\delta R^{2}\right\rangle & =\frac{4}{\left\langle N_{\mathrm{ch}}\right\rangle}\left\langle\delta N_{+}^{2}+\delta N_{-}^{2}-2 \delta N_{+} \delta N_{-}\right\rangle \\
& \approx \frac{4\left\langle\delta Q^{2}\right\rangle}{\left\langle N_{\mathrm{ch}}\right\rangle}
\end{aligned}
$$

where $\left\langle\delta Q^{2}\right\rangle$ is the variance of the net-charge with $Q=$ $N_{+}-N_{-}$being the difference between + ve and - ve particles and $N_{\mathrm{ch}}=N_{+}+N_{-}$being the total number of charged particles measured in a particular phase-space. Assuming the negligible quark-quark interactions, the $D$ is found to be approximately four times smaller in the QGP phase as compared to the hadron gas phase [17]. For uncorrelated pion gas, $D_{\pi}$ is estimated to be 4 and by taking the resonance contributions the value reduces to 3 . For noninteracting massless quarks and gluons $D_{Q G P}$ is found to be a factor of 5 smaller than the $D_{\pi}$. In the constituent quark scenario, it is reported that the $D$-measure value might be $\sim 3.3[19$. The $D$-measure results from ALICE [20] seems to suggest that the hadronization may be due to coalescence which is not the case here, although the fluctuations suggest the existence of non-interacting equilibrated-QGP before the freeze-out. Hence, the measurement of $D$ can be a useful observable to distinguish between the QGP and hadron gas phase [9]. In Ref. 21], it is reported that, there is no significant difference found for $D$ values at SPS [22] and RHIC [23] energies. The $D$ value at $\sqrt{s_{N N}}=200 \mathrm{GeV}$ is observed to be close to the hadron gas prediction [23]. However, recent results from LHC at $\sqrt{s_{N N}}=2.76 \mathrm{TeV}$ [20] show that the measured $D$ value is significantly lower than that measured at SPS and RHIC energies [22, 23]. The value of $D$ at LHC energy approaches the expectation from the QGP phase.

Experimentally, the net-charge fluctuations are studied in terms of dynamical fluctuation measure $\nu_{( \pm, \text {dyn })}$, which is found to be independent of detection efficiencies. The quantity $\nu_{( \pm, \text {dyn })}$ is defined by;

$\nu_{( \pm, \text {dyn })}=\frac{\left\langle N_{+}\left(N_{+}-1\right)\right\rangle}{\left\langle N_{+}\right\rangle^{2}}+\frac{\left\langle N_{-}\left(N_{-}-1\right)\right\rangle}{\left\langle N_{-}\right\rangle^{2}}-2 \frac{\left\langle N_{-} N_{+}\right\rangle}{\left\langle N_{-}\right\rangle\left\langle N_{+}\right\rangle}$

where $\left\langle N_{+}\right\rangle$and $\left\langle N_{-}\right\rangle$are the average number of positive and negative charged particles measured within the detector acceptance. The quantity $\nu_{( \pm, \mathrm{dyn})}$ is a measure of relative correlation of "++", "--" and "+-" charged particle pairs. A positive value of $\nu_{( \pm, \text {dyn })}$ implies the correlation of same charge pairs, where as a negative value indicates the contribution dominated by opposite charge pairs. The $D$ and $\nu_{( \pm, \text {dyn })}$ are related to each other by [17];

$$
\left\langle N_{\mathrm{ch}}\right\rangle \nu_{( \pm, \text {dyn })} \approx D-4
$$

Keeping in mind the importance of the $D$ variable for the conserved number fluctuation, we have estimated the observable in the hadron resonance gas (HRG) model with realistic acceptance effects.

In this work, we demonstrate the effect of different pseudo-rapidity $(|\Delta \eta|)$ intervals and the lower transverse momentum $\left(p_{T}^{\min }\right)$ particle selection threshold on the $D$ in the framework of a HRG model and other heavy-ion models such as heavy-ion jet interaction generator (HIJING) 24] and ultrarelativistic quantum molecular dynamics (UrQMD) 25]. Further, we show the effect of resonance decay on the studied observable. It is important to find an adequate baseline for the conserved quantities particularly for net-charge fluctuations due to contributions from the higher charge states, resonance decays, and also contributions from quantum statistics for lower mass particles such as pions and kaons. Since net-charge fluctuations are dominated by contributions from pions, therefore measured fluctuations are strongly influenced by quantum statistics effects. The present study is an attempt to provide a more realistic baseline comparison to the corresponding experimentally measured quantities.

The paper is organized as follows: In the following section, we discuss the HRG model used in this study as well as the implementation of resonance decays. We also briefly discuss the HIJING and UrQMD heavy-ion models which have been used for comparison with the results obtained from the HRG model. In Sec. III the results for the $D$ observable for different energies, $|\Delta \eta|$ acceptance and effect of the $p_{T}^{\text {min }}$ selection threshold are discussed. The diffusion in rapidity space is studied at different collision energies and presented in the same section. Finally, in Sec. IV we summarize our findings and mention the implication of this work.

\section{THE D-MEASURE FROM DIFFERENT MODEL CALCULATIONS}

In this section, we discuss different models such as HRG, HIJING and UrQMD to understand the $D$ values measured in the experiment. These models provide the proper baseline to compare with the experimental data.

\section{A. Hadron resonance gas model}

The HRG model has been successfully applied to explain the particles produced in heavy-ion collisions from AGS, RHIC, and LHC energies [26 28]. Further, the susceptibilities and their ratios in the hadronic phase calculated in the HRG model reasonably agree with the lattice QCD calculations at lower $\mu_{B}$ values [11]. Several studies have been performed with the HRG model for the fluctuation of conserved quantities, which are considered as a baseline for such measurements 29 35].

The partition function $(Z)$ in the HRG model includes all relevant degrees of freedom of the confined, strongly 
interacting matter and contains all the interactions that result in resonance formation 33]. The heavy-ion experiments covering limited phase space can access only part of the total number of produced particles in the collisions. Hence, the grand canonical ensemble is more appropriate to describe such a scenario [36]. Assuming a thermal system produced in the heavy-ion collisions, the thermodynamic pressure $(P)$ can be written as a sum of the partial pressures of all the considered particle species $i$ which can be baryon $(B)$ or meson $(M)$ at temperature $T$ and chemical potential $\mu_{B}$ :

$$
P\left(T, V, \mu_{i}\right)=\frac{T}{V} \ln Z_{i}
$$

where $\ln Z_{i}=\sum_{M} \ln Z_{i}\left(T, V, \mu_{i}\right)+\sum_{B} \ln Z_{i}\left(T, V, \mu_{i}\right)$ and $\ln Z_{i}$ of individual particles can be written as:

$$
\ln Z_{i}\left(T, V, \mu_{i}\right)= \pm \frac{V g_{i}}{2 \pi^{2}} \int d^{3} p \ln \left\{1 \pm \exp \left[\left(\mu_{i}-E\right) / T\right]\right\}
$$

where $V$ is the volume of the system, $g_{i}$ is the degeneracy factor of the $i$-th particle and the \pm ve signs are for baryons and mesons, respectively. The total chemical potential of the individual particle is $\mu_{i}=$ $B_{i} \mu_{B}+Q_{i} \mu_{Q}+S_{i} \mu_{S}$, where $B_{i}, Q_{i}$, and $S_{i}$ are the baryon, electric charge and strangeness number of the $i$-th particle, with corresponding chemical potentials $\mu_{B}$, $\mu_{Q}$ and $\mu_{S}$, respectively. The collision energy dependence of freeze-out parameters $\left(\mu_{B}\right.$ and $\left.T_{\mathrm{f}}\right)$ is used as given in Ref. 27] and the parametrized $\mu_{Q}$ and $\mu_{S}$ are given in Ref. [33]. The volume element $\left(d^{3} p\right)$ of a particle of mass $m$ in a static fireball can be written as $d^{3} p=p_{T} m_{T} \cosh \eta d p_{T} d \eta d \phi$ and energy $\left(E=m_{T} \cosh \eta\right)$ of the particle, where $m_{T}$ corresponds to the transverse mass of the particle $\left(\sqrt{m^{2}+p_{T}^{2}}\right)$ with $p_{T}, \eta$, and $\phi$ being the transverse momentum, pseudo-rapidity, and azimuthal angle, respectively. One can apply the acceptance cuts on these variables to compare with the experimental results. The $n$-th order generalized susceptibilities $(\chi)$ are written as [34]:

$$
\chi_{i}^{(n)}=\frac{d^{n}\left[P(T, \mu) / T^{4}\right]}{d\left(\mu_{i} / T\right)^{n}} .
$$

For mesons, $\chi_{i}$ can be expressed as;

$$
\begin{aligned}
& \chi_{i, \text { meson }}^{(n)}=\frac{I^{n}}{V T^{3}} \int d^{3} p \sum_{k=0}^{\infty}(k+1)^{n-1} \\
& \times \exp \left\{\frac{-(k+1) E}{T}\right\} \exp \left\{\frac{(k+1) \mu}{T}\right\},
\end{aligned}
$$

and for baryons,

$$
\begin{aligned}
& \chi_{i, \text { baryon }}^{(n)}=\frac{I^{n}}{V T^{3}} \int d^{3} p \sum_{k=0}^{\infty}(-1)^{k}(k+1)^{n-1} \\
& \times \exp \left\{\frac{-(k+1) E}{T}\right\} \exp \left\{\frac{(k+1) \mu}{T}\right\},
\end{aligned}
$$

where $I$ represents either $B_{i}, Q_{i}$ or $S_{i}$ of the $i$ th particle depending on whether the susceptibility $\chi_{i}$ represents for net-baryon, net-electric charge, or net-strangeness. The total generalized susceptibilities will be the sum of susceptibility of mesons and baryons.

The experimentally measured stable particles (pions, kaons, and protons along with their anti-particles) have contributions from primordial as well as from resonance decay. Neutral resonances introduce positive correlations between $N_{+}$and $N_{-}$and hence the decayed daughters from resonances can affect the fluctuation of the final measured particles. The generalized $n$-th order susceptibility for stable particle $i$ can be written as [37, 38];

$$
\chi_{i}^{(n)}=\chi_{i}^{*(n)}+\sum_{R} \chi_{R}^{(n)}\left\langle n_{i}\right\rangle_{R}^{n}
$$

The first term in Eq. 9 corresponds to the contribution from primordial yield and the second term corresponds to the contribution from the resonance particles to stable particles. The summation runs over all the resonance states which contribute to the final particle $i$ and $\left\langle n_{i}\right\rangle_{R}=\sum_{r} b_{r}^{R} n_{i, r}^{R}$ is the average number of particle type $i$ produced from the resonance $R$. Further, $b_{r}^{R}$ is the branching ratio of the $r$-th decay channel of the resonance $R$ and $n_{i, r}^{R}$ is the number of particle $i$ produced in that decay branch. In this study, we have considered the fluctuation in the resonance production and the fluctuation in the produced daughters from the resonance. Detailed discussion on resonance decay can be found in Refs. [37, 38].

\section{B. D-measure using HIJING and UrQMD model}

In the present study, we discuss the $D$ observable calculated using different heavy-ion models. Here we briefly discuss event generators such as HIJING 24] and UrQMD models 25]. HIJING is a perturbative QCD model which produces minijet partons that are later transformed into string fragments that then fragment into hadrons. The cross sections for hard parton scattering are calculated using the leading order in order to account for the higher-order corrections, and a $K$-factor is invoked. The diquark-quark strings with gluon kinks induced by soft gluon radiation are used to calculate the soft contributions. Jet quenching and shadowing can also be treated in this approach [39]. The UrQMD is a microscopic transport approach based on the propagation of constituent quarks and diquarks accompanied by mesonic and baryonic degrees of freedom [25]. It simulates multiple interactions of baryon-baryon, meson-baryon, and meson-meson pairs. The model preserves the conservation of baryon number, electric charge and strangeness number. It also models the baryon-stopping phenomena which is one of the essential features in high-energy heavy-ion collisions particularly at lower collision energies. In this model, the space-time evolution of the fire- 
ball is studied in terms of excitation and fragmentation of color strings, and the formation and decay of hadronic resonances [21, 40].

\section{RESULTS AND DISCUSSION}

The measured fluctuations may get diluted during evolution of the system from hadronization to the kinetic freeze-out because of the diffusion of the charged particles [14]. It is proposed to study the net-charge fluctuations as a function of rapidity interval, which has been explored with the ALICE experiment at LHC by studying $D$ as a function of the pseudorapidity interval $(\Delta \eta)$ [20].

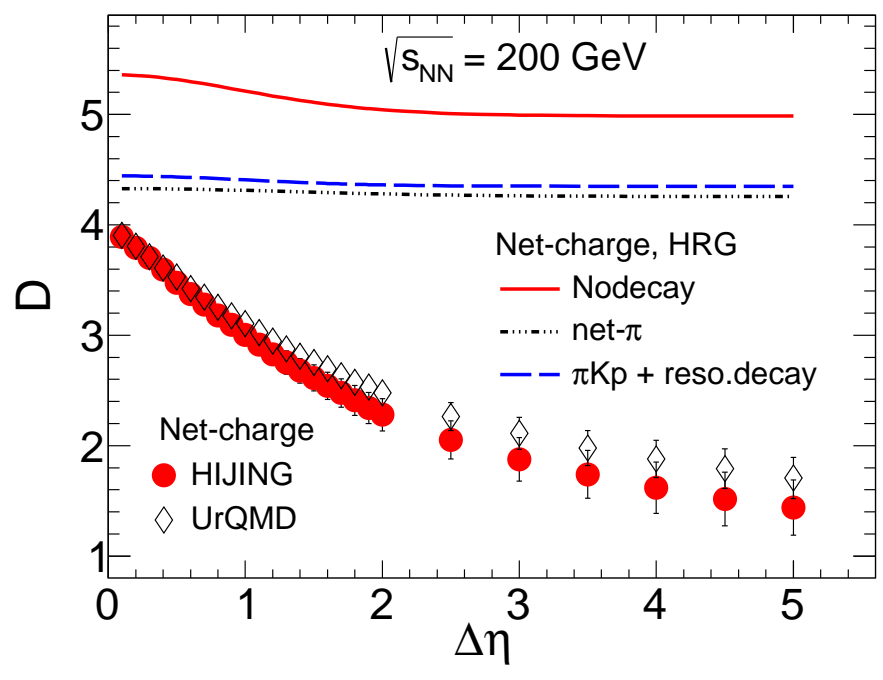

FIG. 1: Fluctuation, $D$, for net-charge as a function of $\Delta \eta$ for $(0 \%-5 \%)$ centrality in $\mathrm{Au}+\mathrm{Au}$ collisions at $\sqrt{s_{N N}}=200 \mathrm{GeV}$ with HIJING and UrQMD models. The $D$ from HRG calculations for net-charge without resonance decay (solid line), with resonance decay (dashed line) and with net-pion (dasheddotted line) are also shown.

It is reported that, the $D$-measure has a decreasing trend with a flattening tendency at larger $\Delta \eta$ values [20].

\section{A. D-measure from different models}

We have investigated this study with HIJING and UrQMD event generators up to large $\Delta \eta$ interval at different $\sqrt{s_{N N}}$. As discussed in the previous section, although both the models are based on different physics inputs, these models have been successfully applied to explain the experimental data at RHIC energies. Figure 1 shows the $D$ as a function of $\Delta \eta$ interval. All the stable charged particles having $p_{T}$ within 0.2 to 5.0 $\mathrm{GeV} / c$ are considered for the analysis. The $D$ values from both the models agree well for all the $\Delta \eta$ intervals within

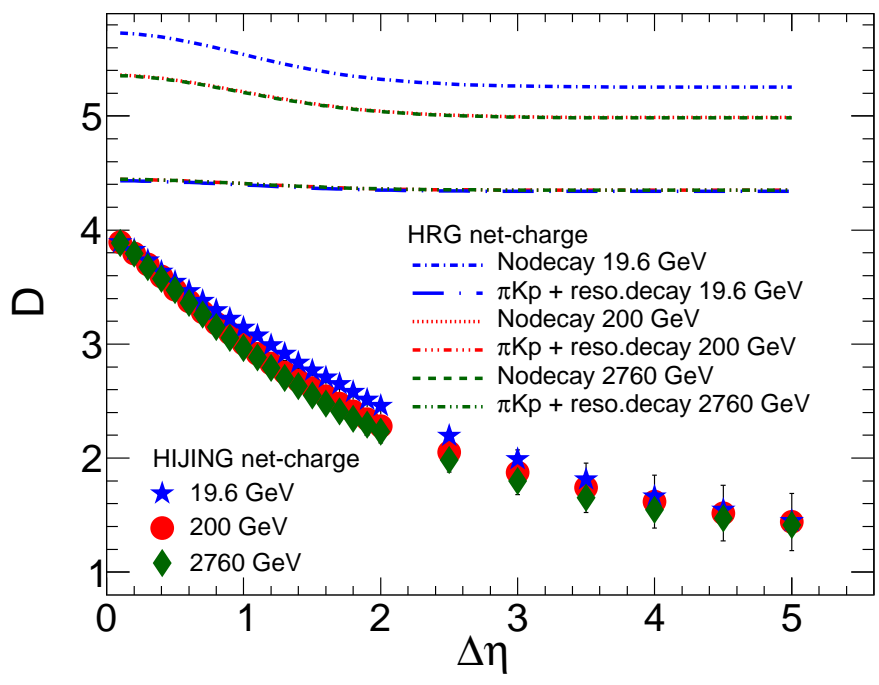

FIG. 2: The $D$ values for net-charge fluctuations as a function of $\Delta \eta$ for $(0 \%-5 \%)$ centrality in $\mathrm{Au}+\mathrm{Au}$ collisions at $\sqrt{s_{N N}}$ $=19.6,200$ and $2760 \mathrm{GeV}$ with the HIJING model. The $D$ from HRG, with and without resonance decay contribution, compared with model calculations at different $\sqrt{s_{N N}}$ energies.

the statistical uncertainties. The $D$ value decreases as a function of $\Delta \eta$. The higher $D$ value at smaller $\Delta \eta$ interval suggests that the correlation is maximum for the smaller $\Delta \eta$ interval which gets diluted at larger intervals. The curvature of $D$ shows a decreasing slope up to higher $\Delta \eta$ interval. This is in contrast to the observation made by ALICE experiment at $\sqrt{s_{N N}}=2.76 \mathrm{TeV}$, which predicts a flattening trend at large $\Delta \eta$ by extrapolation of the fitted curve to the higher $\Delta \eta$ region 20]. The $D$ values from the HRG calculation are compared with the HIJING and UrQMD results. The HRG calculation for net-charge fluctuations is performed within the same kinematic acceptance as done for other models. All the charged hadrons of mass up to $2.5 \mathrm{GeV}$ as listed in the particle data book are considered. The HRG calculations for net-charge fluctuations are performed by considering all charged particles without resonance decay, only primordial pions, and stable charged particles $(\pi, K$, and $p$ ) with resonance decay contributions. Unlike HIJING and UrQMD model results, the HRG calculations do not show $\Delta \eta$ dependence of $D$. In case of no decay of resonances, there is small dependence observed at lower $\Delta \eta$ intervals. However, there is substantial decrease of $D$ value with inclusion of resonance decay contributions as compared to without decay of resonances. Since charge fluctuation is dominated by fluctuations from the pions, we have also compared the $D$ values for primordial pions by taking quantum statistics into account. The $D$ values for net-pions are closer to the results obtained from netcharge fluctuation. The calculation of $D$ from the HRG model will provide a pure thermal baseline as a function $\Delta \eta$. 


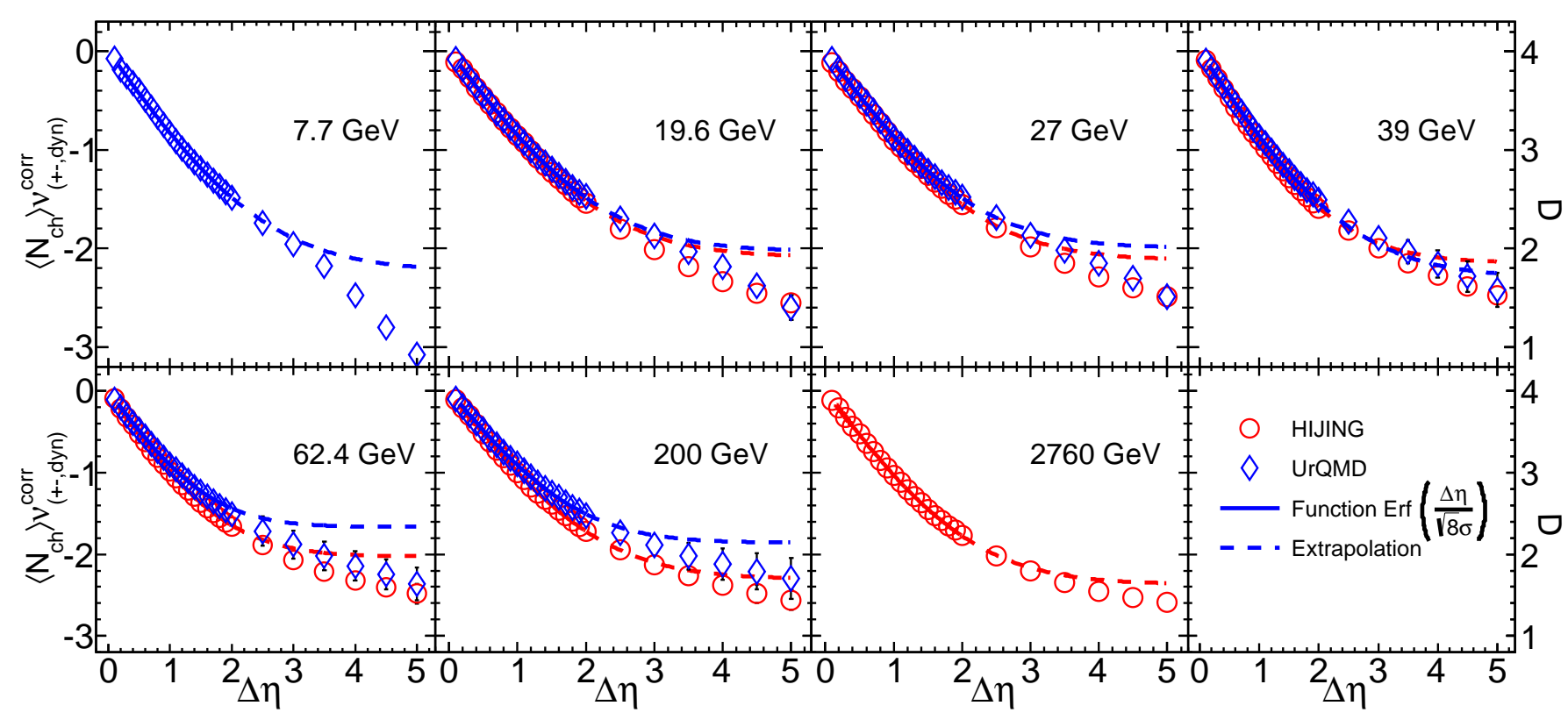

FIG. 3: The $\left\langle N_{c h}\right\rangle \nu_{( \pm, \text {dyn })}^{\text {corr }}$ (left axis) and $D$ (right axis) as a function of $\Delta \eta$ intervals for $(0 \%-5 \%)$ centrality in Au+Au collisions at different $\sqrt{s_{N N}}$. The data points are fitted with the $\operatorname{Erf}(\Delta \eta / \sqrt{8} \sigma)$ up to $\Delta \eta=2.0$ shown in solid line and extrapolation up to higher $\Delta \eta$ intervals is shown in dashed line.

\section{B. Energy dependence of $D$-measure}

The $\Delta \eta$ dependences of the $D$ observable obtained from both HIJING and UrQMD models are very similar. Hence for the energy dependence studies, we consider results from the HIJING model. In order to study the energy dependence of $D$ as a function of $\Delta \eta$ intervals, we have considered simulated events for three different energies $\sqrt{s_{N N}}=19.6,200$ and $2760 \mathrm{GeV}$ using HIJING. Figure 2] shows the $\Delta \eta$ dependence of $D$ of net-charge fluctuations for different energies. The $D$ values are consistently decreasing for all the studied energies up to large $\Delta \eta$ interval. We do not observe flattening behavior at higher $\Delta \eta$ intervals for any of the considered energies as predicted by the extrapolation of experimental data [20]. The $D$ values obtained from the HRG calculations are compared with results from HIJING. In case of without decay of resonances, the HRG result shows a higher value for $19.6 \mathrm{GeV}$ than the other two higher energies and remains constant as a function $\Delta \eta$ intervals, except small dependence at lower $\Delta \eta$ intervals. However, the $D$ values agrees well for all studied energies with inclusion of resonance decay contributions in the HRG calculations.

\section{Extraction of diffusion parameter}

The measured fluctuation signals may get obliterated during the evolution of the system in heavy-ion nuclear collisions because of the diffusion of the charged particles in rapidity space. It is estimated that, the net-charge fluctuations induced by quark gluon plasma hadronization may survive diffusion in the hadronic stage [14]. It is also discussed how much the fluctuations are reduced with the increase of accepted rapidity interval. It is observed from the experimental data at $\sqrt{s_{N N}}=2.76 \mathrm{TeV}$ that the $D$ value has a decreasing slope at lower $\Delta \eta$ intervals and gets flattened at higher $\Delta \eta$ intervals [20]. Figure 3 shows the $\left\langle N_{\mathrm{ch}}\right\rangle \nu_{( \pm, \text {dyn })}^{\text {corr }}$ and $D$ as a function of $\Delta \eta$ interval for $(0 \%-5 \%)$ centrality in $\mathrm{Au}+\mathrm{Au}$ collisions at different $\sqrt{s_{N N}}$ using HIJING and UrQMD models. In a similar way as in Ref. [20], the simulated data points are fitted with the error function, $\operatorname{Erf}(\Delta \eta / \sqrt{8} \sigma)$ representing the diffusion in rapidity space [14]. This accounts for the broadening of the rapidity distributions due to interactions and particle production. The quantity $\sigma$ which characterizes the diffusion at freeze-out is obtained by fitting the $\left\langle N_{\mathrm{ch}}\right\rangle \nu_{( \pm \text {,dyn })}^{\text {corr }}$ values up to $\Delta \eta=2.0$. The results from both HIJING and UrQMD models do not show flattening behavior for $\Delta \eta$ above 1.5. The $\left\langle N_{\mathrm{ch}}\right\rangle \nu_{( \pm, \text {dyn })}^{\text {corr }}$ and $D$ values from the models keep on decreasing even at higher $\Delta \eta$ intervals. Figure 4 shows the comparison of $\left\langle N_{\mathrm{ch}}\right\rangle \nu_{( \pm \text {,dyn })}^{\text {corr }}$ and $D$ as a function of $\Delta \eta$ obtained from the experimental data in Ref. [20] and the model calculations at $\sqrt{s_{N N}}=2760 \mathrm{GeV}$. The experimental data show flattening behavior of the fluctuations at higher $\Delta \eta$ as compared to the HIJING model calculations. The slope of the experimental data and the HIJING model calculations are different, which results in different extrapolated values at higher $\Delta \eta$. With larger uncertainties in the experimental data, it may so happen that the extrapolated values at higher $\Delta \eta$ will follow the experimental mea- 
surements. Further, the HRG calculations do not show $\Delta \eta$ dependence of $D$ in both without and with resonance decay contributions. The experimental data [20] and the present study with HIJING and UrQMD models follow the diffusion trend.

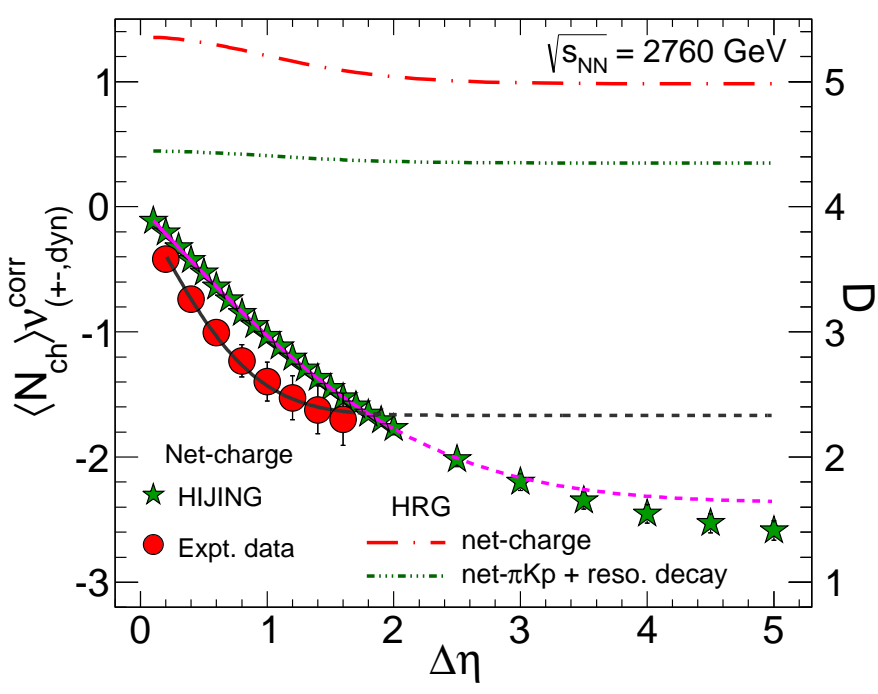

FIG. 4: The $\left\langle N_{c h}\right\rangle \nu_{( \pm \text {,dyn) }}^{\text {corr }}$ (left axis) and $D$ (right axis) as a function of $\Delta \eta$ intervals for $(0 \%-5 \%)$ centrality in $\mathrm{Pb}+\mathrm{Pb}$ collisions at different $\sqrt{s_{N N}}$. The data points are fitted with the $\operatorname{Erf}(\Delta \eta / \sqrt{8} \sigma)$ up to $\Delta \eta=2.0$ shown in solid line and extrapolation up to higher $\Delta \eta$ intervals is shown in dashed line. The $D$ from HRG, with and without resonance decay contribution, compared with the experimental data and HIJING model calculation.

The extrapolation of the fitted curves in Fig. 3 do not explain the $D$ values extracted at higher $\Delta \eta$ intervals particularly at lower energies. Hence, in order to estimate the diffusion parameters from $D$ values, it is important to measure up to higher $\Delta \eta$ intervals. The resulting values of $\sigma$ obtained from the above fit function at different $\sqrt{s_{N N}}$ are shown in Fig. 5a. The collision energy dependence of the $D$ values is shown in Fig [5]. The $D$ values shown in Fig [5] are extracted from two different methods. The values extracted from the simulated data at $\Delta \eta=5.0$ are shown in solid symbols and the $D$ values calculated from the extrapolation of the fitted curve up to higher $\Delta \eta$ at 5.0 are shown in open symbols. The $D$ values estimated using the extrapolation method show higher values as compared to the values from the data points at $\Delta \eta=5.0$ obtained from the models. This can also be observed in Fig. 3 at higher $\Delta \eta$ intervals. However, the data points from the HIJING simulation are in better agreement with the extrapolated curve for $\sqrt{s_{N N}}$ $=2.76 \mathrm{TeV}$.

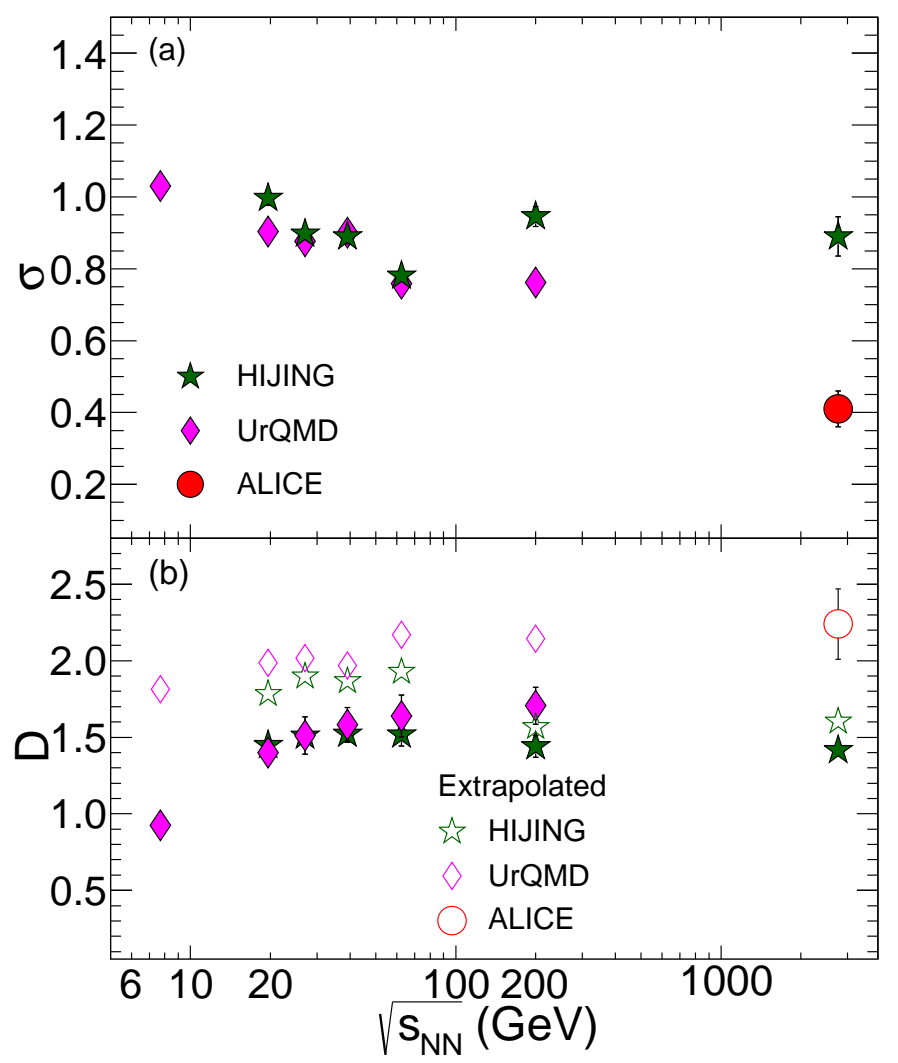

FIG. 5: Collision energy dependence of $\sigma$ (upper panel) which characterizes the diffusion at the freeze-out are calculated using HIJING and UrQMD models for $(0 \%-5 \%)$ centrality in $\mathrm{Au}+\mathrm{Au}$ collisions. The $D$ values as a function of $\sqrt{s_{N N}}$ are shown in the lower panel. The solid symbols are values extracted from the simulated data at $\Delta \eta=5.0$ and the open symbols are extrapolated values of $D$ using the functional form at the same $\Delta \eta$ interval.

\section{Particle dependence of $D$-measure}

The net-charge fluctuation is mostly dominated by the fluctuation contribution from pions. Hence it is important to identify the individual contributions of the stable charged hadrons to the net-charge fluctuation. Figure 6 shows the $D$ of net-charge fluctuations as a function of $\Delta \eta$ interval in $\mathrm{Au}+\mathrm{Au}$ collisions at $\sqrt{s_{N N}}=200 \mathrm{GeV}$ from the HIJING model. The $D$ for net-pions $\left(\pi^{+}-\pi^{-}\right)$, net-kaons $\left(K^{+}-K^{-}\right)$, and net-protons $(p-\bar{p})$ are also shown. In the HIJING model we also observe that the $D$ values for net-charge fluctuations are dominated by netpion fluctuations. The $D$ values of individual net-protons and net-kaons are similar and decrease with increasing $\Delta \eta$ interval. The studied observables for net-charge and net-baryon as a function of $\Delta \eta$ interval calculated in the HRG model are also compared. The net-charge and netbaryon values are compared for calculations with and without resonance decay contributions. Due to the contributions from the resonance decay, the $D$ values are 


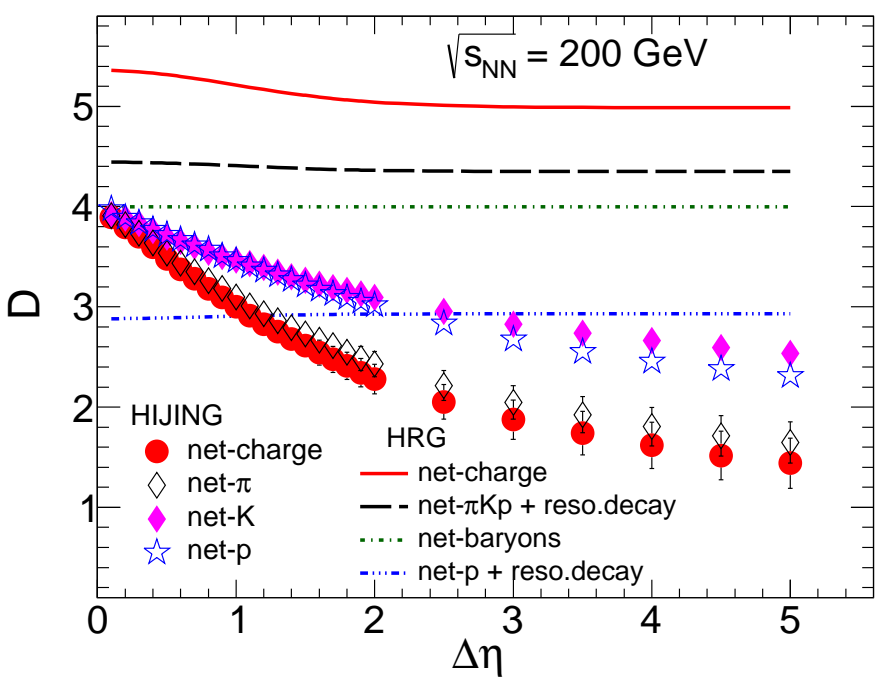

FIG. 6: The $D$ values for net-charge, net-pion, net-kaon, and net-proton fluctuations as a function of $\Delta \eta$ for $(0 \%-5 \%)$ centrality in $\mathrm{Au}+\mathrm{Au}$ collisions at $\sqrt{s_{N N}}=200 \mathrm{GeV}$ with the HIJING model. The $D$ from HRG calculations for net-charge and net-baryon without and with inclusion of resonance decay are compared with model calculations.

substantially smaller when compared to calculations taking all the charged particles or all the baryons. Unlike the results from the HIJING model, the $D$ values for netcharge obtained from the HRG calculations are higher compared to net-baryon, with or without the resonance decay contributions. This may be due to the fact that charged particles with higher electric charge contribute to the higher net-charge fluctuations in HRG.

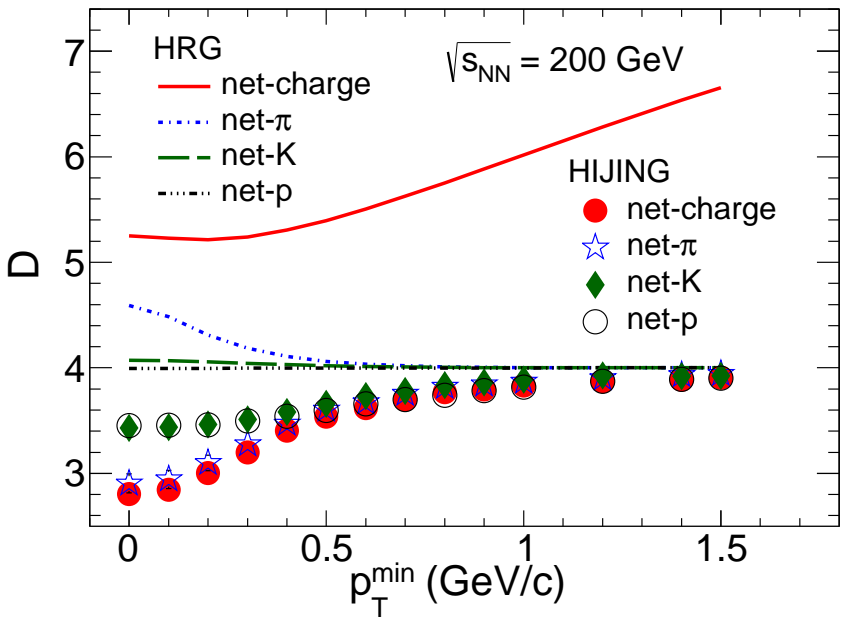

FIG. 7: The lower $p_{T}$ selection range dependence of the $D$ for net-charge, net-pion, net-kaon, and net-proton fluctuations calculated for $(0 \%-5 \%)$ centrality in $\mathrm{Au}+\mathrm{Au}$ collisions at $\sqrt{s_{N N}}=200 \mathrm{GeV}$ with the HIJING model. The $D$ from HRG calculations is compared with HIJING model calculations.

\section{E. Lower $p_{T}$ selection threshold dependence of $D$-measure}

A different experiment might have different lower $p_{T}$ acceptance for particles, hence it is important to study the effect of lower $p_{T}$ selection threshold on the selected charged particles. Figure 7 shows the $p_{T}^{\text {min }}$ selection threshold dependence of $D$ for net-charge, net-pion, netkaon, and net-proton fluctuations in $\mathrm{Au}+\mathrm{Au}$ collisions at $\sqrt{s_{N N}}=200 \mathrm{GeV}$ using the HIJING model. The considered particles are within $|\eta|<0.5$ and maximum $p_{T}$ is $5.0 \mathrm{GeV} / c$. Since the net-charge fluctuations are dominated by net-pion fluctuations, the net-charge and netpion fluctuations are similar and increase with $p_{T}^{\text {min }}$ selection threshold. A saturation trend is observed in $D$ after $p_{T}^{\min } \gtrsim 0.5 \mathrm{GeV} / c$. The lower $p_{T}$ selection threshold has a smaller effect on net-kaon and net-proton fluctuation as compared to net-pion fluctuations. In case of kaons and protons, the mass is already so large that the quantum statistics effect is almost negligible, and thus the result can be regarded as that obtained with Boltzmann approximation. The results from the HIJING model are compared with the $D$ values from the HRG calculations. The net-kaon and net-proton results from the HRG calculations are least affected by the $p_{T}^{m i n}$ selection threshold. The net-pion results are affected at lower $p_{T}$ selection threshold due to the quantum statistics and at higher $p_{T}^{\min }$ selection threshold, the pion momentum distribution can be approximated with Boltzmann statistics. The increasing trend in net-charge fluctuation in HRG calculation is due to the contribution from the resonances with higher electric charge states such as $\Delta^{++}$.

The evolution of net-charge fluctuations with collision energy has been studied by various experiments at SPS [22], RHIC [23], and LHC [20] energies. Figure 8 shows the collision energy dependence of $D$ for net-charge fluctuations in most central $(0 \%-5 \%)$ collisions. The experimental data shows a systematic decrease of $D$ value as one goes from lower to higher collision energies. The experimental measurements are compared with model calculations for net-charge fluctuations. The model calculations are performed within the same acceptance as experimental acceptances. The results from both HIJING and UrQMD models agree with each other and are independent of collision energies. The HRG calculations for net-charge fluctuations with and without inclusion of resonance decay are also shown in Fig. 8 , The $D$ values of net-charge from HRG calculations decrease at lower $\sqrt{s_{N N}}$ and remains constant at higher energies. By including the resonance decay contributions, the $D$ values are independent of $\sqrt{s_{N N}}$, which can be considered as a baseline for comparison with the experimental data. The experimentally measured $D$ values at lower energies are closer to the HRG calculation with inclusion of resonance decay contributions and deviates from the HRG calculation for higher energies. In Ref. [9], it is shown that the $D$ value for hadron gas with resonance decay contributions is $\simeq 3$ and for the QGP phase is $\simeq 1.0-1.5$. The 


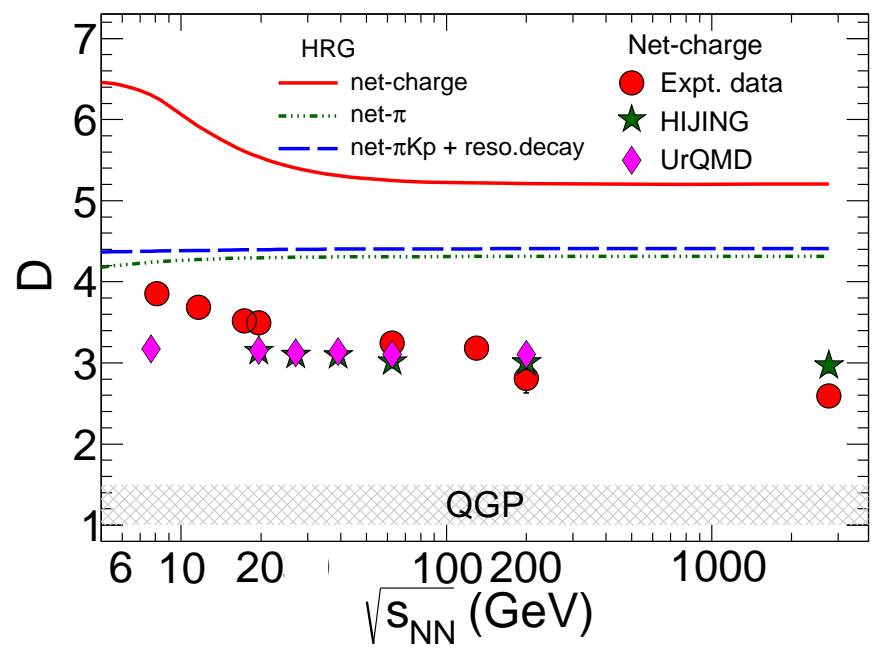

FIG. 8: Collision energy dependence of $D$ for the net-charge fluctuations measured at mid-rapidity in central (0\%-5\%) heavy-ion collisions at SPS [22], RHIC [23], and LHC 20] energies. The model calculations (HIJING, UrQMD and HRG) for different energies are also shown.

ALICE experiment reported the $D$ value of $2.3 \pm 0.22$ for $\Delta \eta=1.6$ at $\sqrt{s_{N N}}=2.76 \mathrm{TeV}[20]$. This value is lower than the results at lower energies and in-between hadron gas and QGP prediction [9]. In the present study with the HRG model, we estimate the $D$ value $\simeq 4.4$ by taking proper kinematic acceptance and resonance decay contributions. This serves as a more realistic baseline for comparison of experimental data in the hadronic phase. Comparing the experimental results from Ref. 20] with the HRG calculation, it can be inferred that the experimentally measured $D$ value at LHC energy is substantially lower than the HRG calculation and closer to those for the expectations in the QGP phase.

\section{SUMMARY}

In summary, we have studied net-charge fluctuation using the $D$-measure observable within the ambit of HI-
JING, UrQMD and HRG models. The $D$ values are estimated up to higher $\Delta \eta$ intervals. A stronger dependence of $D$ value is observed at lower $\Delta \eta$ intervals and a decreasing trend continues up to higher $\Delta \eta$ intervals. Results from both the HIJING and UrQMD models agrees with each other up to $\sqrt{s_{N N}}=200 \mathrm{GeV}$. The HRG calculations with and without resonance decay contributions are also compared. We do not observe $\Delta \eta$ acceptance dependence of the studied observable in the HRG calculation except at lower energies when resonance decay contributions are not considered. However, there is a significant effect of resonance decay contributions to the $D$ observable. We also studied the $D$ as a function of $\Delta \eta$ interval for net-charge and individual stable particles. The net-charge fluctuation is dominated by the contribution from net-pion fluctuation. Both net-charge and net-pion fluctuations decrease with increasing $\Delta \eta$ interval. The $D$ values for net-kaons and net-protons also decrease with increasing $\Delta \eta$ interval with less steeper slope when compared to net-charge and net-pion fluctuations. The effect of lower $p_{T}$ selection threshold on the $D$ observable is also studied in HIJING and HRG models. The net-charge and net-pion fluctuations are more affected by $p_{T}^{\min }$ selection threshold and increase with $p_{T}$ cutoff. The net-kaon and net-proton results are least affected by the $p_{T}$ cutoff both in HIJING and HRG calculations. The dissipation of signal during the evolution of the fireball from the hadronization to freeze-out has been estimated for different energies by fitting the $D$-measure as a function of the $\Delta \eta$ interval with the error function. The extrapolation of the fitted curve does not explain the $D$ values calculated at higher $\Delta \eta$ intervals from HIJING and UrQMD models. It is to be noted that we observe more discrepancy at lower $\sqrt{s_{N N}}$ as compared to LHC energy. We have also studied the $D$ for different collision energies for most central $(0 \%-5 \%)$ collisions. The results obtained from the model calculations are independent of $\sqrt{s_{N N}}$. The experimental measurement at $\sqrt{s_{N N}}=2760 \mathrm{GeV}$ is significantly lower than the HRG calculation and closer to QGP prediction. This study provides a more realistic baseline for comparison of experimental data and will be useful for other upcoming experiments also.
[1] M. A. Stephanov, K. Rajagopal and E. V. Shuryak, Phys. Rev. Lett. 81, 4816 (1998).

[2] M. G. Alford, K. Rajagopal and F. Wilczek, Phys. Lett. B 422, 247 (1998).

[3] M. A. Stephanov, Phys. Rev. Lett. 76, 4472 (1996).

[4] Y. Aoki, G. Endrodi, Z. Fodor, S. D. Katz and K. K. Szabo, Nature 443, 675 (2006).

[5] R. D. Pisarski and F. Wilczek, Phys. Rev. D 29, 338 (1984).

[6] S. Ejiri, Phys. Rev. D 78, 074507 (2008).

[7] M. A. Stephanov, K. Rajagopal and E. V. Shuryak, Phys. Rev. D 60, 114028 (1999).
[8] M. Asakawa, U. W. Heinz and B. Muller, Phys. Rev. Lett. 85, 2072 (2000).

[9] S. Jeon and V. Koch, Phys. Rev. Lett. 83, 5435 (1999).

[10] S. Ejiri, F. Karsch and K. Redlich, Phys. Lett. B 633, 275 (2006).

[11] A. Bazavov, H. T. Ding, P. Hegde, O. Kaczmarek, F. Karsch, E. Laermann, S. Mukherjee and P. Petreczky et al., Phys. Rev. Lett. 109, 192302 (2012).

[12] J. Adams et al. [STAR Collaboration], Phys. Rev. C 68, 044905 (2003).

[13] P. Alba, W. Alberico, R. Bellwied, M. Bluhm, V. Mantovani Sarti, M. Nahrgang and C. Ratti, Phys. Lett. B 
738, 305 (2014).

[14] M. A. Aziz and S. Gavin, Phys. Rev. C 70, 034905 (2004).

[15] E. V. Shuryak and M. A. Stephanov, Phys. Rev. C 63, 064903 (2001).

[16] Y. Hatta and M. A. Stephanov, Phys. Rev. Lett. 91, 102003 (2003) Erratum: [Phys. Rev. Lett. 91, 129901 (2003)].

[17] S. Jeon and V. Koch, In *Hwa, R.C. (ed.) et al.: Quark gluon plasma* 430-490 hep-ph/0304012.

[18] C. Pruneau, S. Gavin and S. Voloshin, Phys. Rev. C 66, 044904 (2002).

[19] A. Bialas, Phys. Lett. B 532, 249 (2002).

[20] B. Abelev et al. [ALICE Collaboration], Phys. Rev. Lett. 110, 152301 (2013).

[21] M. Bleicher, S. Jeon and V. Koch, Phys. Rev. C 62, 061902 (2000).

[22] H. Sako et al. [CERES/NA45 Collaboration], J. Phys. G 30, S1371 (2004).

[23] B. I. Abelev et al. [STAR Collaboration], Phys. Rev. C 79, 024906 (2009).

[24] X. N. Wang and M. Gyulassy, Phys. Rev. D 44, 3501 (1991).

[25] M. Bleicher et al., J. Phys. G 25, 1859 (1999).

[26] P. Braun-Munzinger, K. Redlich and J. Stachel, In *Hwa, R.C. (ed.) et al.: Quark Gluon Plasma 3, 491-599 (2004).

[27] J. Cleymans, H. Oeschler, K. Redlich and S. Wheaton, Phys. Rev. C 73, 034905 (2006).

[28] A. Andronic, P. Braun-Munzinger, K. Redlich and
J. Stachel, J. Phys. G 38, 124081 (2011).

[29] D. K. Mishra, P. K. Netrakanti and B. Mohanty, Phys. Rev. C 94, 054906 (2016).

[30] V. V. Begun, M. I. Gorenstein, M. Hauer, V. P. Konchakovski and O. S. Zozulya, Phys. Rev. C 74, 044903 (2006).

[31] F. Becattini, A. Keranen, L. Ferroni and T. Gabbriellini, Phys. Rev. C 72, 064904 (2005).

[32] J. Fu, Phys. Lett. B 722, 144 (2013).

[33] F. Karsch and K. Redlich, Phys. Lett. B 695, 136 (2011).

[34] P. Garg, D. K. Mishra, P. K. Netrakanti, B. Mohanty, A. K. Mohanty, B. K. Singh and N. Xu, Phys. Lett. B 726, 691 (2013).

[35] P. Rau, J. Steinheimer, S. Schramm and H. Stocker, Phys. Lett. B 733, 176 (2014).

[36] V. Koch, Chapter of the book "Relativistic Heavy Ion Physics", R. Stock (Ed.), Springer, Heidelberg, 2010, p. 626-652. (Landolt-Boernstein New Series I, v. 23).

[37] D. K. Mishra, P. Garg, P. K. Netrakanti and A. K. Mohanty, Phys. Rev. C 94, 014905 (2016).

[38] M. Nahrgang, M. Bluhm, P. Alba, R. Bellwied and C. Ratti, Eur. Phys. J. C 75, 573 (2015).

[39] Q. H. Zhang, V. Topor Pop, S. Jeon and C. Gale, Phys. Rev. C 66, 014909 (2002).

[40] B. Sharma, M. M. Aggarwal, N. R. Sahoo and T. K. Nayak, Phys. Rev. C 91, 024909 (2015). 